\title{
Advances in detector-integrated filter coatings for the far ultraviolet
}

John Hennessy, April Jewell, Michael Hoenk, Shouleh Nikzad

John Hennessy, April D. Jewell, Michael E. Hoenk, Shouleh Nikzad, "Advances in detector-integrated filter coatings for the far ultraviolet," Proc. SPIE 11821, UV, X-Ray, and Gamma-Ray Space Instrumentation for Astronomy XXII, 118211A (24 August 2021); doi: 10.1117/12.2595524

SPIE Event: SPIE Optical Engineering + Applications, 2021, San Diego, California, United States 


\title{
Advances in detector-integrated filter coatings for the far ultraviolet
}

\author{
John Hennessy*a, April D. Jewell ${ }^{\text {a }}$, Michael E. Hoenk ${ }^{\text {a }}$, Shouleh Nikzad ${ }^{\text {a }}$ \\ a Jet Propulsion Laboratory, California Institute of Technology, 4800 Oak Grove Drive, Pasadena, \\ CA, USA 91109
}

\begin{abstract}
We report on the continued development of multilayer optical coatings on back-illuminated silicon imaging sensors in order to enhance the functionality of such systems at ultraviolet wavelengths. This includes the development metaldielectric filter structures to enable solar-blind operation, and graded thickness coatings to tune the spatial response of a detector system to the dispersion of a spectrometer. Such systems can maintain the high internal quantum efficiency afforded by the delta-doping process utilized at NASA JPL, while also providing long-wavelength rejection or a spatially optimized efficiency (or both). We present the characterization of CCD and CMOS image sensors incorporating these processes, and describe the atomic layer deposition coating processes. Such detectors are currently being developed for ground-based high energy physics applications as well as NASA orbital astrophysics instruments operating at wavelengths shorter than $220 \mathrm{~nm}$.
\end{abstract}

Keywords: ultraviolet, detector, filter, atomic layer deposition, thin film, spectroscopy

\section{DETECTOR INTEGRATED METAL-DIELECTRIC FILTERS}

The development of back-illuminated, delta-doped (and superlattice-doped) silicon imaging sensors at JPL has led to sensor systems that can operate with $100 \%$ internal quantum efficiency (QE) across the ultraviolet and visible spectrum.[1] Throughput at the focal plane is then limited only by reflection losses which may be less than $35 \%$ at visible wavelengths, but peak at more $70 \%$ in the UV. Dielectric anti-reflection coatings can recover a portion of these losses but cannot effectively cover the full UV/visible spectrum with a single coating due to the changing refractive index of silicon over this range.[2]

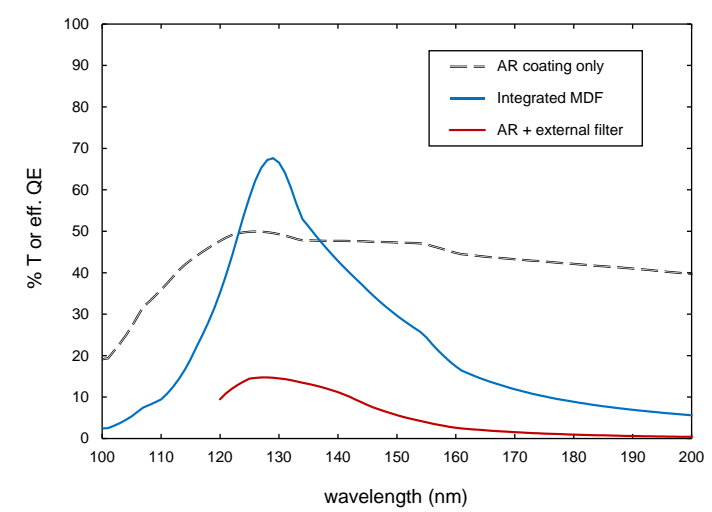

Figure 1. The modeled performance of a directly integrated metal-dielectric filter (MDF) versus a simple anti-reflection coating, and an anti-reflection coating plus stand-alone filter optimized for a bandpass near $130 \mathrm{~nm}$.

*john.j.hennessy@jpl.nasa.gov

UV, X-Ray, and Gamma-Ray Space Instrumentation for Astronomy XXII, edited by Oswald H. Siegmund, Proc. of SPIE Vol. 11821, 118211A · (c) 2021 SPIE

CCC code: $0277-786 X / 21 / \$ 21 \cdot$ doi: $10.1117 / 12.2595524$ 
For many UV sensing applications it also desirable to reduce the detector response at longer wavelengths. Although this can be achieved by external filtering, the efficiency of such an approach is greatly reduced at targets wavelengths less than $200 \mathrm{~nm}$, due to materials limitations. In the far ultraviolet (FUV, $\lambda \approx 90-200 \mathrm{~nm}$ ) such filters typically utilize a metal-dielectric structure of alternating layers of aluminum and transparent dielectric spacers. Because silicon itself is an effective UV reflector, there are significant throughput advantages in integrating these coatings directly on a device as highlighted in Figure 1.[3,4] In these calculations the modeled transmission of the filter coating is equivalent to the predicted external QE of the detector due to the $100 \%$ internal QE afforded by the delta-doping process. As shown in Figure 2, these filters have been developed at JPL for a number of applications including high energy physics detectors,[5,6] and astrophysics instruments such as the SPARCS CubeSat.[7] Continued development of similar filters is also supporting instruments concepts on several NASA Astrophysics Explorer-class missions.
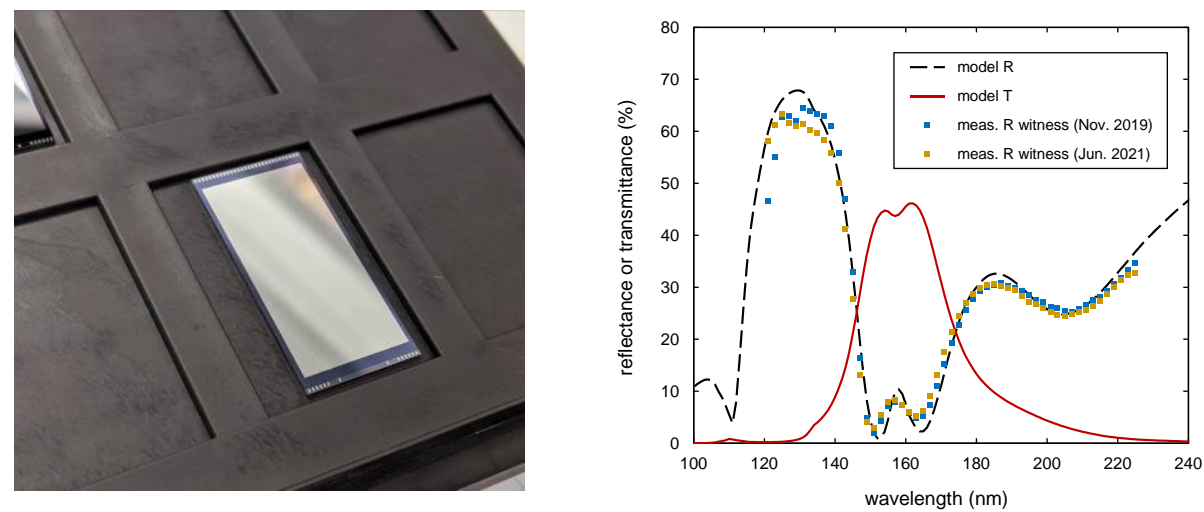

Figure 2. (left) A delta-doped Teledyne e2v CCD with an integrated metal dielectric bandpass filter. The filter structure combines three $\mathrm{Al}$ layers deposited by evaporation with four $\mathrm{AlF}_{3}$ layers deposited by atomic layer deposition (ALD). (right) The measured reflectance of witness samples to the device on the left, and the best-fit model reflectance and predicted transmittance. Little change in witness reflectance is observed for long duration storage.

The aluminum layers in these filters are deposited by evaporation, and the dielectric spacer layers utilize a number of atomic layer deposition (ALD) processes developed at JPL.[8-10] FUV performance requires the use of metal fluoride materials like $\mathrm{MgF}_{2}$ and $\mathrm{AlF}_{3}$ to be used as the spacer layers. In some cases, these materials are associated with aging concerns due to environmental instabilities. Figure 2 also shows that witness samples monitored over more than one year show little change in measured reflectance. Samples were stored in a nitrogen-purged dry box environment over this time frame. Note that the modeled transmission prediction (effective QE) includes absorption losses expected in the multilayers. Custom thin-film vacuum chambers have also been developed at JPL to optimize the combination of these filters with back-illuminated, delta-doped detectors.

\section{GRADED THICKNESS COATINGS BY ATOMIC LAYER DEPOSITION}

The ALD method is often utilized in our optical coating processes to take advantage of properties related to film thickness uniformity. For coatings on imaging sensors, it is often desirable to have absolute uniformity in coating thickness so that the ultimate response of the detector system is also spatially uniform. However, for spectroscopic applications it may be advantageous to change the thickness of a potential coating to match the spatial dispersion of light across a device. Such an approach is common at visible and infrared wavelengths through the use of wedge filters or linear variable filters (LVF). However, the most common implementation utilizes a stand-alone LVF placed in front of the focal plane.[11] These graded optical coatings are typically produced by physical vapor deposition (PVD) processes like evaporation and shuttering via mechanical masking or shuttering of the line-of-sight PVD process. 
Because ALD is not a line-of-sight process, physical shuttering or masking of the substrate cannot be used to create thin films with a graded lateral thickness. Since our existing process development, qualification, and materials analysis is built around the use of ALD, it would be advantageous to engineer a graded thickness with the same technique in order to optimize detector response for spectroscopic applications. As shown in Figure 3, we have explored a technique to force ALD into a non-uniform mode by depositing films inside a shallow vertical cavity. The geometry of this configuration limits the extent to which ALD precursors can diffuse into the cavity and therefore yields a gradual roll-off in film thickness as function of depth into the cavity. Functional tests were performed by separating two silicon wafers with a precision stainless steel shim. This general approach is analogous to the use of lateral high aspect ratio structures that have been previously used to study fundamental aspects of ALD film growth kinetics.[12]
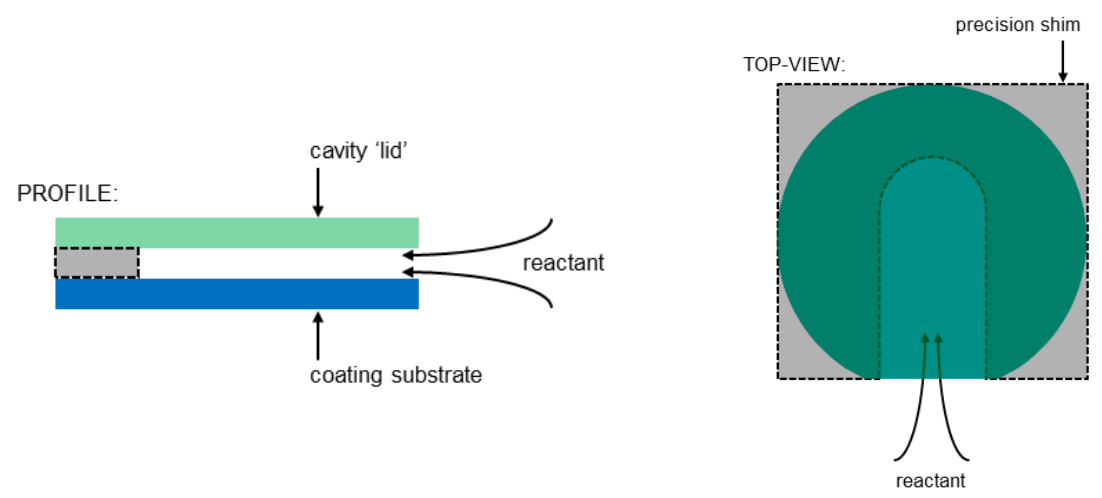

Figure 3. Deposition into a shallow cavity limits the amount of reactant that can penetrate before the next ALD half cycle begins. Functional testing of this concept was performed by placing a precision stainless steel shim between two silicon wafers.

This technique has the possible advantage that it requires no moving parts inside the vacuum chamber in order to produce this change in film thickness. One constraint is that for a given set of process conditions (e.g. cavity height, working pressure, precursor dose time) the resulting profile is fixed as a function of position along the length of cavity. However, because ALD is a 'digital' method, individual cycles within the full process can be mixed with different process conditions. As shown in Figure 4, one parameter that can be used to alter the lateral profile is working process pressure, which is determined in our system by the Ar purge gas flow rate. Variation of this pressure can tune the spatial extent of the grading over most of the length of the cavity. The final desired slope or spatial profile (linear or otherwise) can be achieved by superposition of these profiles relative to the fraction of total cycles in the full ALD process.

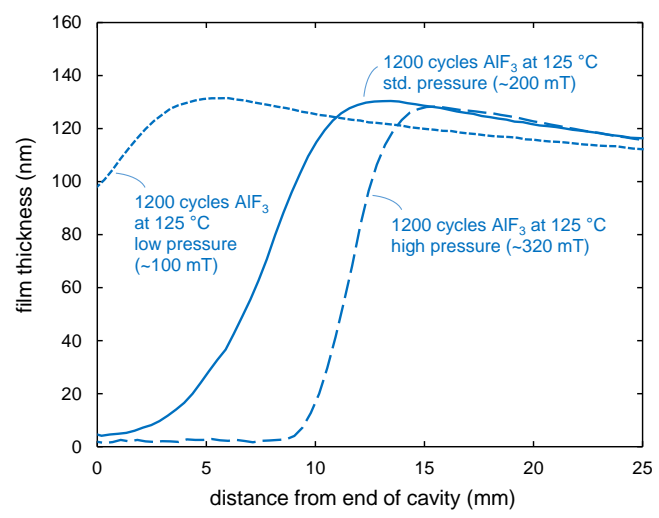

Figure 4. Measured variation in $\mathrm{AlF}_{3}$ film thickness as a function of distance into the cavity. The penetration depth can be varied by altering the height of the cavity or the working pressure of the process as shown here. Arbitrary positive slopes can be engineered by combining ALD cycles at different pressures. 


\section{IMPROVED THROUGHPUT FOR ULTRAVIOLET SPECTROSCOPY}

Extension of this method to practical spectroscopic examples was first considered at near UV and visible wavelengths as shown in Figure 6. Here, a graded thickness coating was considered to maximize transmission in the range of 300$1000 \mathrm{~nm}$. Planar coatings can achieve a maximum average transmission of $\sim 90 \%$ in this range,[2] but a graded three layer coating of $\mathrm{HfO}_{2} / \mathrm{Al}_{2} \mathrm{O}_{3} / \mathrm{HfO}_{2}$ is projected to achieve greater than $99 \%$ transmission across the entire band. In this case, only the middle $\mathrm{Al}_{2} \mathrm{O}_{3}$ layer is graded in thickness and the two $\mathrm{HfO}_{2}$ layers are uniform.

The initial demonstration of this approach discussed in the previous section utilized an $\mathrm{AlF}_{3}$ ALD process with trimethyaluminum and hydrogen fluoride as process precursors. In principle, the technique can be extended to any thermal ALD process. The design in Figure 6 was implemented with a graded layer of $\mathrm{Al}_{2} \mathrm{O}_{3}$ using trimethylaluminum and water as reactants and required a recalibration of profiles like those in Figure 4 to establish the appropriate process control points for the new combination of reactants.
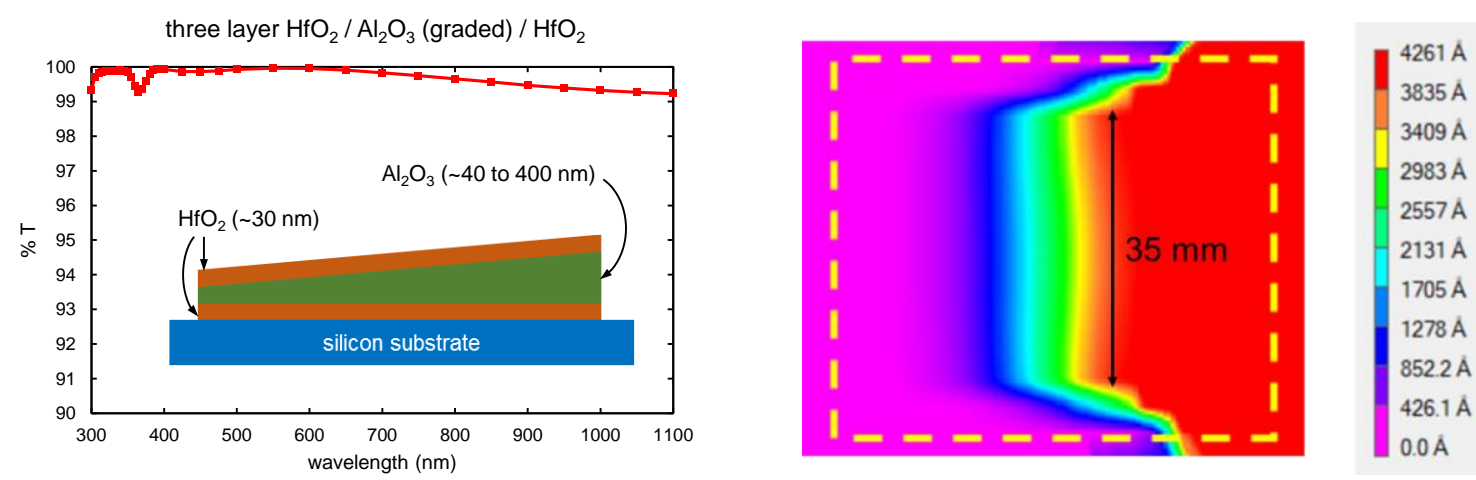

Figure 6. (left) The modeled transmission (red curve) of the three-layer AR coating on Si shown in the inset. (right) The measured spatial variation in $\mathrm{Al}_{2} \mathrm{O}_{3}$ thickness for this three-layer coating deposited by the ALD cavity method on a Si wafer. The region of approximately uniform vertical profile is $35 \mathrm{~mm}$ wide and corresponds to the physical opening of the cavity used.

The full three-layer stack was deposited onto a silicon wafer and characterized by spatial reflectance spectroscopy mapping also shown in Figure 6. From this mapping, the approximate thickness of the middle $\mathrm{Al}_{2} \mathrm{O}_{3}$ layer was extracted as a function of $x$ and $y$ position. The vertical extent of the graded profile is decided by the width of the cavity used, in this case a laterally graded profile with a width of $35 \mathrm{~mm}$. This is already sufficiently large to coat a number of technologically relevant scientific imaging sensors. The same coating procedure can be used by just replacing the bottom substrate with the required silicon detector substrate. In the current implementation we are limited to performing this coating process at the die level. Larger cavities can be utilized to create patterns with larger width, constrained only by the overall reactor geometry. The absolute performance achieved in our three-layer testing coating was verified by inspecting individual spectra of the reflectance mapping at various points along the $x$ direction of the sample. Figure 7 shows that a minimum reflectance of $<1 \%$ is obtained across the spectral range of interest. 


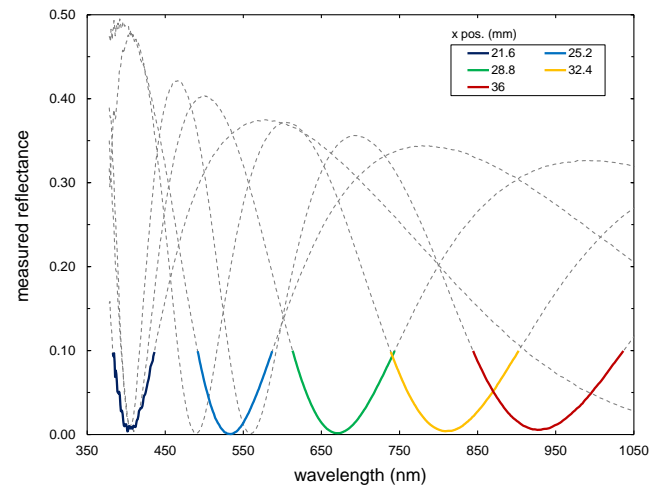

Figure 7. The measured spectral reflectance of the three-layer stack at various points along the graded direction. A minimum reflectance of $<1 \%$ is achieved for each of the measured spectra with the minimum regions of each individual spectrum highlighted for clarity.

Finally, this graded layer concept can be combined with metal-dielectric structures to extend the performance of such coatings into the FUV. Figure 8 shows a calculated composite transmission (or effective detector QE) of a three-layer metal-dielectric optimized for 100-350 nm utilizing only a single graded layer of $\mathrm{AlF}_{3}$ as the starting thin film. The final two layers of $\mathrm{Al} / \mathrm{AlF}_{3}$ are uniform. This could be easily realized experimentally by combining the results of Figure 4 with existing MDF processes described in $\S 1$. Such a coating is predicted to outperform the bare Si reflection limit by a significant margin, and can outperform any one possible AR coating material across a broad wavelength range. $[2,13]$ This approach potentially comes with additional advantages related to order sorting and red rejection depending on the spectrometer requirements. Additional improvement in transmittance can be obtained by grading additional layers in the stack. Additional red rejection can also be obtained with additional layer complexity in the full stack.

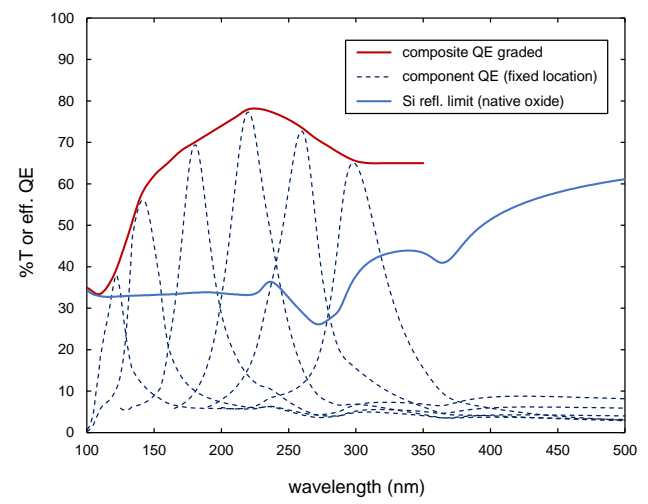

Figure 8. Metal-dielectric structures that incorporate graded thickness can significantly improve throughput over bare $\mathrm{Si}$ devices in the FUV. The modeled QE shown here is for a simple three-layer filter with a single graded layer of $\mathrm{AlF}_{3}$ capped with uniform layers of $\mathrm{Al}$ and $\mathrm{AlF}_{3}$.

\section{ACKNOWLEDGEMENTS}

The research described in this paper was carried out at the Jet Propulsion Laboratory, California Institute of Technology, under a contract with the National Aeronautics and Space Administration. Government sponsorship is acknowledged. 


\section{REFERENCES}

[1] Nikzad, S., Jewell, A. D., Hoenk, M. E., Jones, T., Hennessy, J., Goodsall, T., Carver, A., Shapiro, C., Cheng, S. R., Hamden, E. and Kyne, G., "High efficiency UV/optical/NIR detectors for large aperture telescopes and UV explorer missions: development of and field observations with delta-doped arrays," J. Astron. Telesc. Instrum. Syst. 3(3), 036002 (2017).

[2] Jewell, A. D., Hennessy, J., Hoenk, M. E. and Nikzad, S., "Wide band antireflection coatings deposited by atomic layer deposition," Proc. SPIE 8820, 88200Z (2013).

[3] Hennessy, J., Jewell, A. D., Hoenk, M. E., and Nikzad, S., "Metal-dielectric filters for solar-blind silicon ultraviolet detectors," Appl. Opt. 54(11), 3507-3512 (2015).

[4] Hennessy, J., Jewell, A. D., Hoenk, M. E., Hitlin, D., McClish, M., Carver, A. G., Jones, T. J. and Nikzad, S., "Materials and process development for the fabrication of far ultraviolet device-integrated filters for visible-blind $\mathrm{Si}$ sensors," Proc. SPIE 10209, 102090P (2017).

[5] Hoenk, M. E., Nikzad, S., Carver, A. G., Jones, T. J., Hennessy, J., Jewell, A. D., Sgro, J., Tsur, S., McClish, M., and Farrell, R., "Superlattice-doped silicon detectors: progress and prospects," Proc. SPIE 9154, 915413 (2014).

[6] Hitlin, D., Kim, J. H., Trevor, J., Hennessy, J., Hoenk, M., Jewell, A., Farrell, R. and McClish, M., "An APD for the detection of the fast scintillation component of $\mathrm{BaF}_{2}$," IEEE Trans. Nucl. Sci. 63(2), 513-515 (2016).

[7] Jewell, A., Hennessy, J., Jones, T., Cheng, S., Carver, A., Ardila, D., Shkolnik, E., Hoenk, M. and Nikzad, S., "Ultraviolet detectors for astrophysics missions: a case study with the star-planet activity research cubesat (SPARC)," Proc. SPIE 10709, 107090C (2018).

[8] Hennessy, J., Jewell, A. D., Greer, F, Lee, M. C., and Nikzad, S., "Atomic layer deposition of magnesium fluoride via bis(ethylcyclopentadienyl)magnesium and anhydrous hydrogen fluoride," J. Vac. Sci. Technol. A 33, 01A125 (2015).

[9] Hennessy, J., Jewell, A. D., Balasubramanian, K., and Nikzad, S., "Ultraviolet optical properties of aluminum fluoride thin films deposited by atomic layer deposition,” J. Vac. Sci. Technol. A 34, 01A120 (2016).

[10] Hennessy, J., and Nikzad, S., "Atomic Layer Deposition of Lithium Fluoride Optical Coatings for the Ultraviolet," Inorganics 6(2), 46 (2018).

[11] Demro, J. C., Hartshorne, R., Woody, L. M., Levine, P. A. and Tower, J. R., "Design of a multispectral, wedge filter, remote-sensing instrument incorporating a multiport, thinned, CCD area array," Proc. SPIE 2480, 280-286 (1995).

[12] Ylilammi, M., Ylivaara, O. M. and Puurunen, R. L., "Modeling growth kinetics of thin films made by atomic layer deposition in lateral high-aspect-ratio structures," J. Appl. Phys. 123(20), 205301 (2018).

[13] Hamden, E. T., Jewell, A. D., Shapiro, C. A., Cheng, S. R., Goodsall, T. M., Hennessy, J., Hoenk, M. E., Jones, T., Gordon, S., Ong, H. R. and Schiminovich, D., "Charge-coupled devices detectors with high quantum efficiency at UV wavelengths,” J. Astron. Telesc. Instrum. Syst. 2(3), 036003 (2016). 